\title{
Long intergenic non-protein-coding RNA 01446 facilitates the proliferation and metastasis of gastric cancer cells through interacting with the histone lysine-specific demethylase LSD1
}

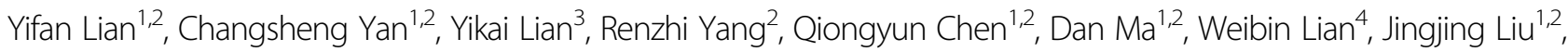
Chengyan Luo ${ }^{5}$, Jianlin Ren ${ }^{1,2}$ and Hongzhi $X^{1,2}$

\begin{abstract}
Growing evidences illustrated that long non-coding RNAs (IncRNAs) exhibited widespread effects on the progression of human cancers via various mechanisms. Long intergenic non-protein-coding RNA 01446 (LINC01446), a 3484-bp ncRNA, is known to locate at chromosome 7p12.1. However, its biological functions and specific action mechanism in gastric cancer (GC) are still unclear. In our study, LINC01446 was proved to be markedly upregulated in GC tissues relative to the normal tissues, and positively correlated with the poor survival of GC patients. The multivariate Cox regression model showed that LINC01446 functioned as an independent prognostic factor for the survival of GC patients. Functionally, LINC01446 facilitated the proliferation and metastasis of GC cells. Moreover, RNA-seq analysis demonstrated that LINC01446 knockdown primarily regulated the genes relating to the growth and migration of GC. Mechanistically, LINC01446 could widely interact with histone lysine-specific demethylase LSD1 and recruit LSD1 to the Ras-related dexamethasone-induced 1 (RASD1) promoter, thereby suppressing RASD1 transcription. Overall, these findings suggest that LINC01446/LSD1/RASD1 regulatory axis may provide bona fide targets for anti-GC therapies.
\end{abstract}

\section{Introduction}

Gastric cancer (GC), a main factor in causing cancerrelated mortalities all over the world, is one of the most common gastrointestinal malignancies in $\mathrm{China}^{1-3}$. Until 2015, GC was considered as the second most common cancer following lung cancer in China based on the latest cancer statistic data ${ }^{1}$. Although the diagnosis and therapies for GC have greatly improved, there are still high recurrence and metastasis rates ${ }^{4}$. Moreover, these

\footnotetext{
Correspondence: Chengyan Luo (betteryuan66@163.com) or

Jianlin Ren (renjianl@xmu.edu.cn) or Hongzhi Xu (xuhongzhi@xmu.edu.cn)

'Department of Gastroenterology, Zhongshan Hospital, Xiamen University, Xiamen, Fujian, PR China

${ }^{2}$ Institute for Microbial Ecology, School of medicine, Xiamen University, Xiamen, Fujian, PR China

Full list of author information is available at the end of the article

These authors contributed equally: Yifan Lian, Changsheng Yan, Yikai Lian

Edited by I. Amelio
}

improvements are often limited because the related molecular mechanisms underlying the occurrence and development of GC remain unclear ${ }^{5}$. Thus, it is critical to understand the potential action mechanism of $\mathrm{GC}$ for discovering novel therapies for GC.

Transcriptome studies indicated that more than $70 \%$ of human genomes could be transcribed to RNAs, and most of them were non-coding ${ }^{6}$. In the recent years, some studies on GC action mechanism revealed that long noncoding RNAs (lncRNAs), the epigenetic regulators at transcriptional or posttranscriptional levels, exhibited crucial effects on the proliferation, apoptosis, and metastasis of GC cells, and drug-resistance ${ }^{7-9}$. In addition, other studies indicated that lncRNAs could also serve as potential therapeutic targets for $\mathrm{GC}$, and might be diagnostic and prognostic biomarkers for $\mathrm{GC}^{7,9,10}$. Specifically, low expression of LINC01133 suppressed the

\section{(c) The Author(s) 2020}

(c) (i) Open Access This article is licensed under a Creative Commons Attribution 4.0 International License, which permits use, sharing, adaptation, distribution and reproduction cc. in any medium or format, as long as you give appropriate credit to the original author(s) and the source, provide a link to the Creative Commons license, and indicate if changes were made. The images or other third party material in this article are included in the article's Creative Commons license, unless indicated otherwise in a credit line to the material. If material is not included in the article's Creative Commons license and your intended use is not permitted by statutory regulation or exceeds the permitted use, you will need to obtain permission directly from the copyright holder. To view a copy of this license, visit http://creativecommons.org/licenses/by/4.0/. 
proliferation and metastasis of GC cells via regulating APC expressions and $W n t / \beta$-catenin signals ${ }^{11}$; lncRNA GMAN was related to the metastasis of GC and could promote ephrin A1 translation via competitively binding to GMAN-AS ${ }^{12}$; and lncRNA HOXC-AS3 could promote the occurrence of GC via regulating HDAC5 at transcriptional level ${ }^{13}$. These studies together indicate that as an important kind of non-coding RNAs, IncRNAs exhibit wide effects on regulating the occurrence and development of GC. However, the related functions and specific regulatory mechanism of lncRNAs in GC need further studies to provide potential therapeutic targets for GC.

LINC01446 has been reported in glioblastoma ${ }^{14}$. However, it is unclear whether LINC01446 exerts similar function in GC tumorigenesis. In our study, LINC01446 expression in GC was detected to understand the function and potential mechanism of LINC01446 regulating the proliferation and metastasis of GC cells. The results demonstrated that the increased LINC01446 expression in GC was related to the metastasis of GC and shorter survival time of GC patients. It was also found that LINC01446 knockdown obviously reduced the proliferation and migration of GC cells. Moreover, LINC01446 was confirmed to downregulate the Rasrelated dexamethasone-induced 1 (RASD1) expression at transcriptional level through recruiting the activated histone lysine-specific demethylase LSD1 to the promoter area of RASD1, thereby accelerating the proliferation and metastasis of GC cells.

\section{Results}

\section{LINC01446 is upregulated in GC and related to poor prognosis}

As shown at https://www.ncbi.nlm.nih.gov/gene/? term $=$ LINC01446, human LINC01446 is located at chromosome 7 and includes eight transcripts. LINC01446-v1, an isoform of LINC01446, is consisted of six exons and highly conservative in mammals (Fig. 1a, b). Subsequently, rapid amplification of cDNA ends was performed to identify the full sequence of LINC01446 in SGC7901 cells according to the sequence archived in the RefSeq database of NCBI (full exact length 3484 bp, as shown in Fig. S1a). The analysis for coding potential obviously demonstrated that all isoforms of LINC01446 might lack the proteincoding ability (Fig. 1c). The GTEx data available at https:// www.gtexportal.org/ illustrated that there was a low expression of LINC01446 in normal tissues (Figs. 1d and S1b). Moreover, TCGA pan-cancer analysis revealed that LINC01446 was most significantly upregulated in GC among all types of cancers (Fig. 1e, f). Interestingly, according to the analysis of TCGA data, the expression level of LINC01446 was significantly higher in both of the intestinal type and mixed type of GC compared with that of normal tissues (Fig. S1c). More importantly, the in situ hybridization assay demonstrated that LINC01446 was expressed in GC tissues, but not in the normal tissues (Fig. 1g). Correspondingly, subsequent quantitative real-time PCR (qRT-PCR) analysis in our study revealed that LINC01446 expression was much higher in GC tissues than in the normal tissues (Fig. 1h).

To further assess the key roles LINC01446 played, we investigated the relationship between the clinicopathological characteristic of GC patients and LINC01446 expression. At first, these 100 patients were assigned into low $(n=50)$ and high LINC01446 expression $(n=50)$ groups based on the median value (Fig. 1i). The results showed that the high-expressed LINC01446 in GC patients was positively related to the advanced TNM and distal metastasis (Table 1). Moreover, Kaplan-Meier analysis demonstrated that the high-expressed LINC01446 was also related to the reduced overall survival (OS) of patients (median DFS times: 4.69 vs. 48.72 months, $P<0.001$; median OS times: 6.69 vs. 49.72 months, $P<0.001$ ) (Fig. 1 j, $k$ ), which was consistent with the results from the Kaplan-Meier analysis for TCGA-STAD database (median OS times: 22.03 vs. 70.00 months, $P=0.0065$ ) (Fig. 1l, m). Moreover, Multivariate Cox analysis further demonstrated that the high-expressed LINC01446 was independently related to the reduced OS (DFS, hazard ratio $=4.928 ; 95 \%$ CI: $2.671-9.090 ; P<0.001$; OS, hazard ratio $=4.446 ; 95 \%$ CI: 2.409-8.206; $P<0.001$ ) (Table 2 ). In summary, our findings together indicated that LINC01446 overexpression is an independent prognostic indicator for GC patients and exhibits key effects on GC progression.

\section{LINC01446 facilitates the proliferation and metastasis of GC cells in vitro}

Given that there was a strong relationship between LINC01446 expression in GC and worse prognosis of GC patients, we further explored whether LINC01446 facilitates the proliferation, invasion, and migration of GC cells. Following detecting LINC01446 expressions in five GC cells and GES-1 cells, we interfered LINC01446 expressions in the SGC7901 and BGC823 cells that had high LINC01446 expression using si-LINC01446, and overexpressed LINC01446 in the MGC803 cells that had low LINC01446 expression (Fig. 2a-c). As showed in Fig. $2 \mathrm{~d}-\mathrm{g}$, MTT and colony formation assays showed that LINC01446 interference significantly disrupted the proliferation and colony formation of SGC7901/BGC823 cells. On the contrary, LINC01446 overexpression facilitated the cell proliferation in vitro. Similarly, ethynyldeoxyuridine (EdU)/DAPI immunostaining supported this finding (Figs. $2 \mathrm{~h}$ and S2a). In addition, apoptosis was identified as a key factor contributing to GC cell growth, so we conducted flow cytometry to detect the apoptosis level. The results demonstrated that LINC01446 knockdown by si-LINC01446 significantly increased the percentage of apoptotic cells (Fig. 2i), whereas excessive 


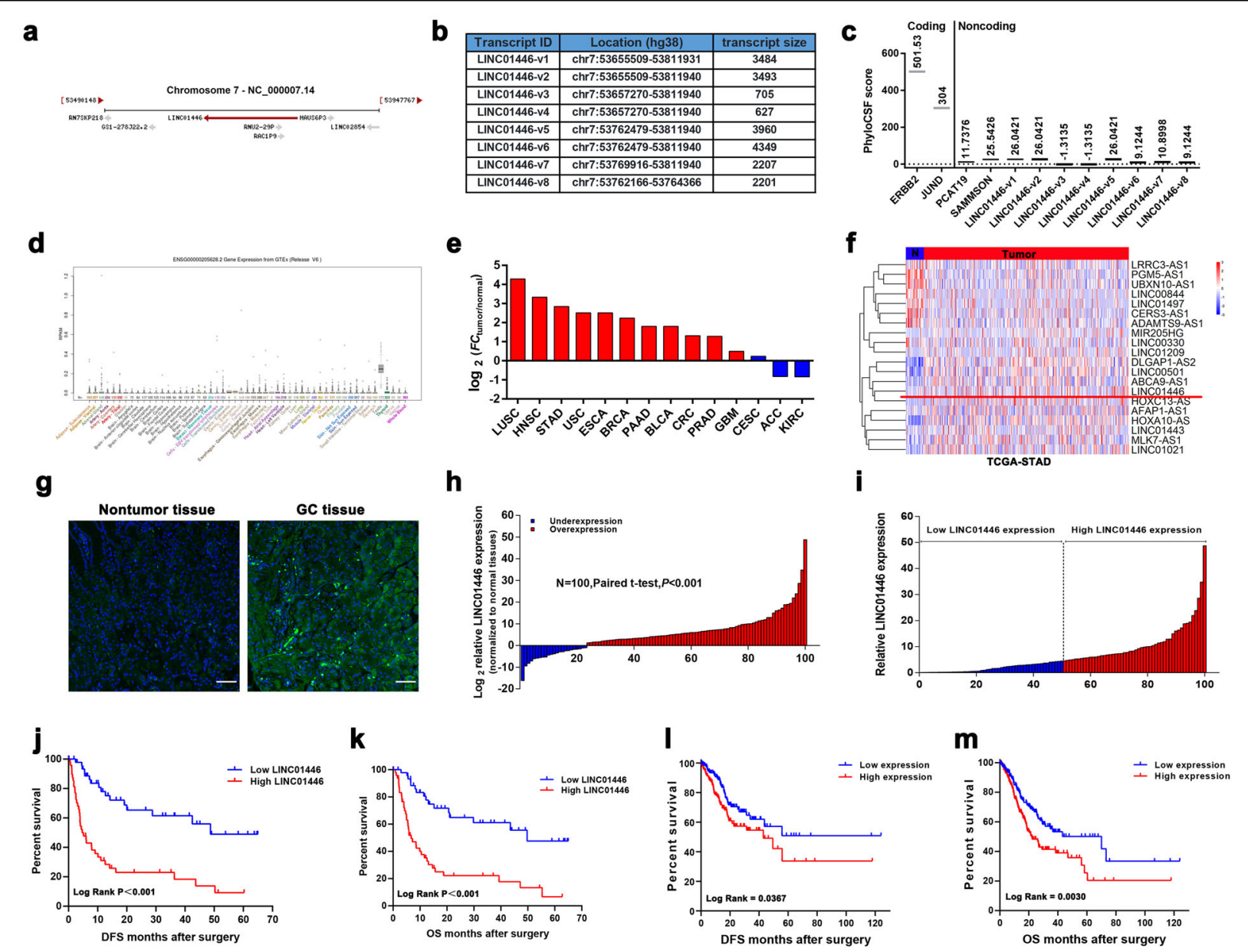

Fig. 1 LINC01446 expression is upregulated in GC tissues and related to the poor prognosis of GC patients. a Schematic annotation for LINC01446 genomic locus. Arrows represented the orientation of transcript. b The list of the human genomic location from IncRNA LINC01446 family. c The maximum cerebrospinal fluid scores of LINC01446 and other known coding and non-coding RNAs were assessed by using PhyloCSF analysis. d LINC01446 expression profile in normal tissues was obtained from UCSC Genome Browser database. e Fold change (FC) of LINC01446 expression (tumor/adjacent normal tissues) in multiple types of cancers from TCGA. $\mathbf{f}$ LINC01446 expression in GC from TCGA database. $\mathbf{g}$ Expression of LINC01446 was detected by using FISH. The green represented positive signal. Scale bar: $80 \mu \mathrm{m}$. $\mathbf{h}$ Analysis for LINC01446 mRNA expressions in 100 matched GC and normal tissues. i GC patients were assigned into two groups following the LINC01446 expression. j, k Kaplan-Meier analysis for disease-free survival and overall survival following the LINC01446 mRNA expression level. I, $\mathbf{m}$ Kaplan-Meier analysis for disease-free survival and overall survival following the LINC01446 mRNA expression level from TCGA database. $\mathbf{h}$, i Student's $t$ test. $\mathbf{j}-\mathbf{m}$ Log-rank test. ${ }^{*} P<0.05$. n.s. not significant.

LINC01446 weakened the percentage of apoptotic cells (Fig. S2b). TUNNEL staining analysis verified the antiapoptotic character of LINC01446 (Fig. S2c). Moreover, wound-healing and transwell assays illustrated that LINC01446 interference markedly suppressed the invasiveness and migration of SGC7901 and BGC823 cells (Fig. 2j, k); in contras, the invasiveness and migration were observably increased depending on the overexpression of LINC01446 in MGC803 cells (Fig. S2d, e). To further evaluate the anticancer effect of si-LINC01446 and confirm our observation in GC cell lines, we interfered LINC01446 in primary GC cells that were isolated from tumor tissues from two GC patients (Fig. 3a). The clinical information about these two patients were listed in Table S4. As expected, the results of MTT and transwell assays demonstrated that the viability and migration of primary GC cells obviously decreased following LINC01446 knockdown (Fig. 3b, c). Correspondingly, the mRNA levels of P15, P16, P21, P27, KLF2 also markedly increased when LINC011446 was interfered (Fig. 3d). Collectively, our data indicated that si-LINC01446 exhibited oncogenic effects on markedly suppressing the proliferation and metastasis of GC cells and the proper regulation for its activity might be a promising strategy for GC treatment. 
Table 1 Correlation between LINC01446 expression and clinicopathologic characteristics of patients with GC $(n=$ 100).

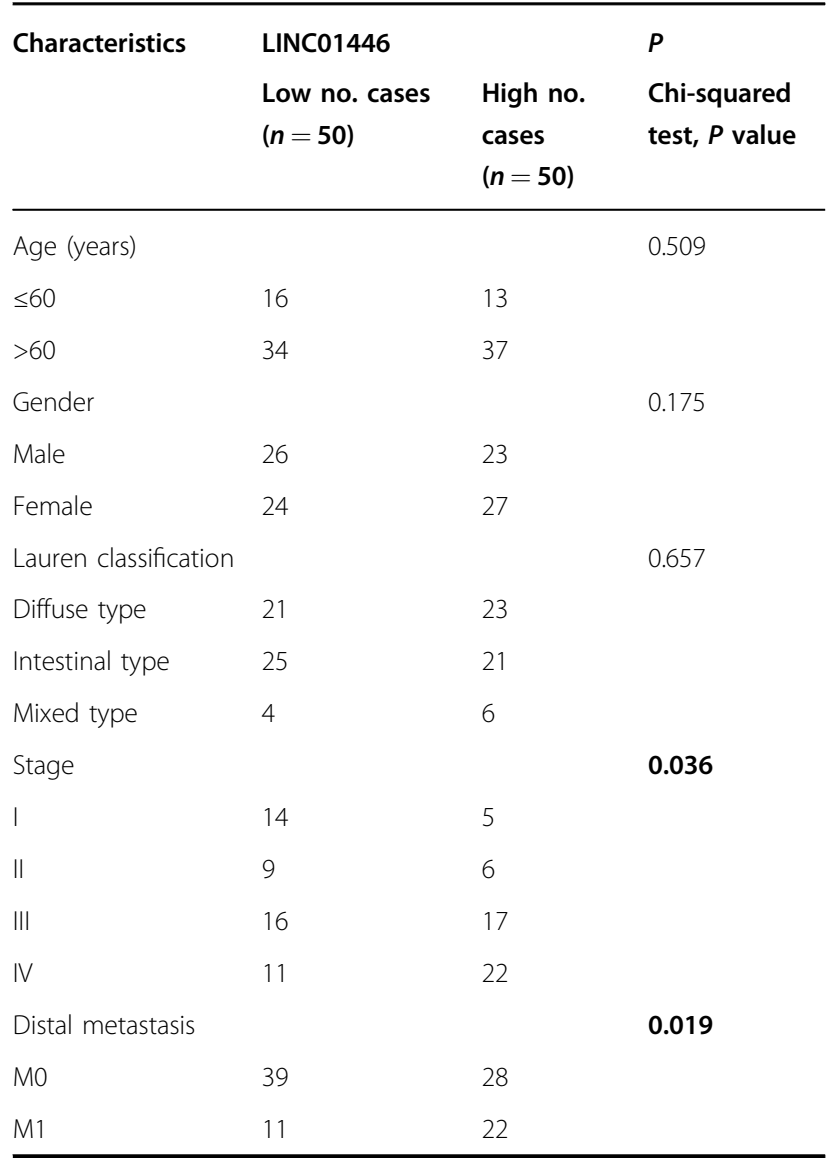

Significant $P$ values are shown in bold font.

LINC01446 facilitates the tumor growth and metastasis of GC in vivo

To further evaluate the effects LINC01446 on the tumor growth of GC, the stably transfected SGC7901 cells with sh-LINC01446 or empty vectors were subcutaneously injected into BALB/c mice, and the tumor growth was measured. The results showed that the tumors were smaller following LINC01446 knockdown than those without LINC01446 knockdown (Fig. 4a). Accordingly, the volume and weight of tumors also obviously decreased in sh-LINC01446 group relative to the empty vector group (Fig. 4b, c). qRT-PCR result demonstrated that the LINC01446 mRNA expression in the tumor tissues with sh-LINC01446 cell transfection was lower than that with control cell transfection (Fig. 4d). Moreover, immunohistochemistry (IHC) showed that the expressions of proliferation marker Ki-67 and proliferating cell nuclear antigen (PCNA) significantly reduced in the shLINC01446-transfected SGC7901 cells relative to the control cells (Fig. 4e).
To further explore the roles LINC01446 plays in the metastasis of GC in vivo, an in vivo mouse model with lung metastasis was constructed. As requested, the stably transfected SGC7901 cells with sh-LINC01446 or empty vectors were injected into the tail veins of six mice, then the metastatic nodules on the surfaces of their lungs were calculated after 6 weeks. The results demonstrated that the LINC01446 interference markedly reduced the numbers of the metastatic nodules (Fig. 4f, g). This finding was further verified by the subsequent examination for the whole lung and the H\&E staining for lung tissues (Fig. 4h). Next, the metastatic lesions in lung sections were also detected by $\mathrm{H} \& \mathrm{E}$ staining, and the result indicated that the percentage of the lung areas with metastatic lesions was much lower in the sh-LINC01446-transfected group than in the empty vector-transfected group (Fig. 4i). In addition, cell adhesion marker fibronectin1 (FN1) expression was also detected using IHC staining, demonstrating that the FN1 expression obviously decreased in the lungs transfected by sh-LINC01446 cells (Fig. 4j).

In summary, all the above data confirmed that the knockdown of LINC01446 could markedly suppress the tumor growth and metastasis of GC, which further validated the oncogenic roles LINC01446 played in GC.

\section{LINC01446 interacts with the histone lysine-specific demethylase LSD1}

Growing evidences indicated that different biological roles IncRNAs played greatly depended on their subcellular localizations ${ }^{15,16}$. As Fig. $5 \mathrm{a}, \mathrm{b}$ showed, LINC01446 expression in the nucleus was more than that in the cytosol of SGC7901 and BGC823 cells, suggesting that LINC01446 may transcriptionally exhibit main regulatory effects. LSD1, an epigenetic, can specifically promote H3K4me2 demethylations, thereby regulating gene transcription. Therefore, we subsequently employed bioinformatics analysis to investigate whether LINC01446 binds to LSD1. The results showed that RF and SVM Classifier scores were more than 0.5 (Fig. 5c), suggesting that LINC01446 is likely to bind to LSD1. To verify this finding, RNA immunoprecipitation (RIP) assay was conducted and showed that LINC01446 could highly bind to LSD1 in SGC7901 and BGC823 cells (Fig. 5d). Next, RNA pull-down assay was also conducted by using LSD1 antibody to further confirm the relationship between LINC01446 and LSD1. The results demonstrated that the labeled LINC01446 RNA, but an antisense LINC01446, specifically retrieved LSD1 from both SCG7901 and BGC823 cell extracts (Fig. 5e). Furthermore, qRT-PCR analysis demonstrated that there were no changes in LSD1 expressions following the knockdown or overexpression of LINC01446 (Fig. 5f, g), and no change in LINC01446 expression was observed after the knockdown of LSD1 (Fig. 5h). These data together indicate that 
Table 2 Univariate and multivariate Cox regression analysis of LINC01446 and survival in patients with GC.

\begin{tabular}{|c|c|c|c|c|c|c|}
\hline \multirow[t]{2}{*}{ Variables } & \multicolumn{3}{|c|}{ Univariate analysis } & \multicolumn{3}{|c|}{ Multivariate analysis } \\
\hline & $P$ value & HR & $95 \% \mathrm{Cl}$ & $P$ value & HR & $95 \% \mathrm{Cl}$ \\
\hline \multicolumn{7}{|l|}{ OS } \\
\hline Age $(\leq 60,>60)$ & 0.995 & 1.000 & $0.979-1.022$ & 0.960 & 0.999 & $0.977-1.022$ \\
\hline Gender (male, female) & 0.125 & 0.636 & $0.357-1.134$ & 0.441 & 0.784 & $0.421-1.457$ \\
\hline Lauren classification & 0.154 & 0.720 & $0.459-1.131$ & 0.021 & 0.576 & $0.361-0.919$ \\
\hline TNM stage (I/II, III/IV) & $<0.001$ & 2.610 & $1.843-3.696$ & $<0.001$ & 2.547 & $1.775-3.655$ \\
\hline Tumor invasion depth (T1/T2, T3/T4) & 0.064 & 1.283 & $0.985-1.671$ & 0.440 & 0.895 & $0.677-1.185$ \\
\hline Lymph node metastasis (NO, N1) & 0.003 & 1.418 & $1.125-1.786$ & 0.760 & 0.952 & $0.693-1.308$ \\
\hline Distant metastasis (yes, no) & $<0.001$ & 4.400 & $2.432-7.959$ & 0.991 & 1.007 & $0.297-3.413$ \\
\hline LINC01446 expression & $<0.001$ & 5.730 & $3.106-10.568$ & $<0.001$ & 4.446 & $2.409-8.206$ \\
\hline \multicolumn{7}{|l|}{ DFS } \\
\hline Age $(\leq 60,>60)$ & 0.993 & 1.000 & $0.979-1.022$ & 0.946 & 1.001 & $0.978-1.024$ \\
\hline Gender (male, female) & 0.122 & 0.626 & $0.351-1.116$ & 0.371 & 0.752 & $0.403-1.403$ \\
\hline Lauren classification & 0.153 & 0.721 & $0.460-1.028$ & 0.020 & 0.576 & $0.362-0.917$ \\
\hline TNM stage (I/II, III/IV) & $<0.001$ & 2.594 & $1.834-3.670$ & $<0.001$ & 2.566 & $1.786-3.687$ \\
\hline Tumor invasion depth $(\mathrm{T} 1 / \mathrm{T} 2, \mathrm{~T} 3 / \mathrm{T} 4)$ & 0.069 & 1.275 & $0.981-1.657$ & 0.371 & 0.881 & $0.667-1.163$ \\
\hline Lymph node metastasis (NO, N1) & 0.002 & 1.433 & $1.137-1.806$ & 0.983 & 0.996 & $0.698-1.421$ \\
\hline Distant metastasis (yes, no) & $<0.001$ & 4.346 & $2.405-7.851$ & 0.845 & 1.113 & $0.379-3.267$ \\
\hline LINC01446 expression & $<0.001$ & 6.126 & $3.324-11.289$ & $<0.001$ & 4.928 & $2.671-9.090$ \\
\hline
\end{tabular}

$\mathrm{HR}>1$, risk for death increased; $\mathrm{HR}<1$, risk for death reduced. Significant $P$ values are shown in bold font.

OS overall survival, DFS disease-free survival, TNM tumor node metastasis, HR hazard ratio, $\mathrm{Cl}$ confidence interval.

LINC01446 binds to LSD1 but has no effects on LSD1 expression, suggesting that LINC01446 may regulate the proliferation and metastasis of GC in transcriptional level through its physical interaction with LSD1.

LSD1 acts as an oncogene in facilitating the proliferation and migration of GC cells

LSD1 was also proved to be associated with the poor prognoses of a variety of tumors ${ }^{17,18}$. Our data demonstrated that the LINC01446 expression was higher in GC cells relative to GES-1 cells (Fig. 5i). To further explore the roles LSD1 plays in the progression of $\mathrm{GC}$, we performed LSD1 knockdown by transfecting si-LSD1 into SGC7901 and BGC823 cells, and then their knockdown efficiencies were evaluated using qRT-PCR and western blotting (Fig. 5j, k). Next, MTT and colony formation assays were carried out to evaluate the roles LSD1 played in the proliferation of GC cells. As expected, LSD1 knockdown significantly suppressed the proliferation and colony formation of SGC7901 and BGC823 cells (Fig. 5l, $\mathrm{m})$. Subsequent transwell assay also showed that LINC01446 knockdown markedly reduced GC cell migration (Fig. 5n). Moreover, further RNA sequencing analysis for TCGA stomach and normal tissues illustrated that LSD1 expression markedly enhanced in GC tissues relative to the normal tissues (Fig. 5o). To further confirm this result, the LSD1 expression in 100 matched GC and normal tissues used in Fig. 1h was measured, and the result indicated that LSD1 was overexpressed in GC tissues (Fig. 5p). All the findings demonstrated that LSD1 could facilitate the proliferation and migration of GC cells, which suggests that LSD1 functions as an important oncogene for GC.

\section{LINC01446 epigenetically suppresses RASD1 transcription by binding to LSD1}

To further explore the potential action mechanism of LINC01446 promoting tumorigenesis, the gene expression profiles for RNA sequencing in the LINC01446-knockdown SGC7901 cells and control cells were analyzed by using gene ontology (GO) analysis and gene set enrichment analysis (GSEA) (Fig. 6a). The GO analysis demonstrated that the most significant biological processes included cell cycle, cell adhesion, and cell junction (Fig. 6b). The GSEA illustrated that gene sets were markedly associated with 


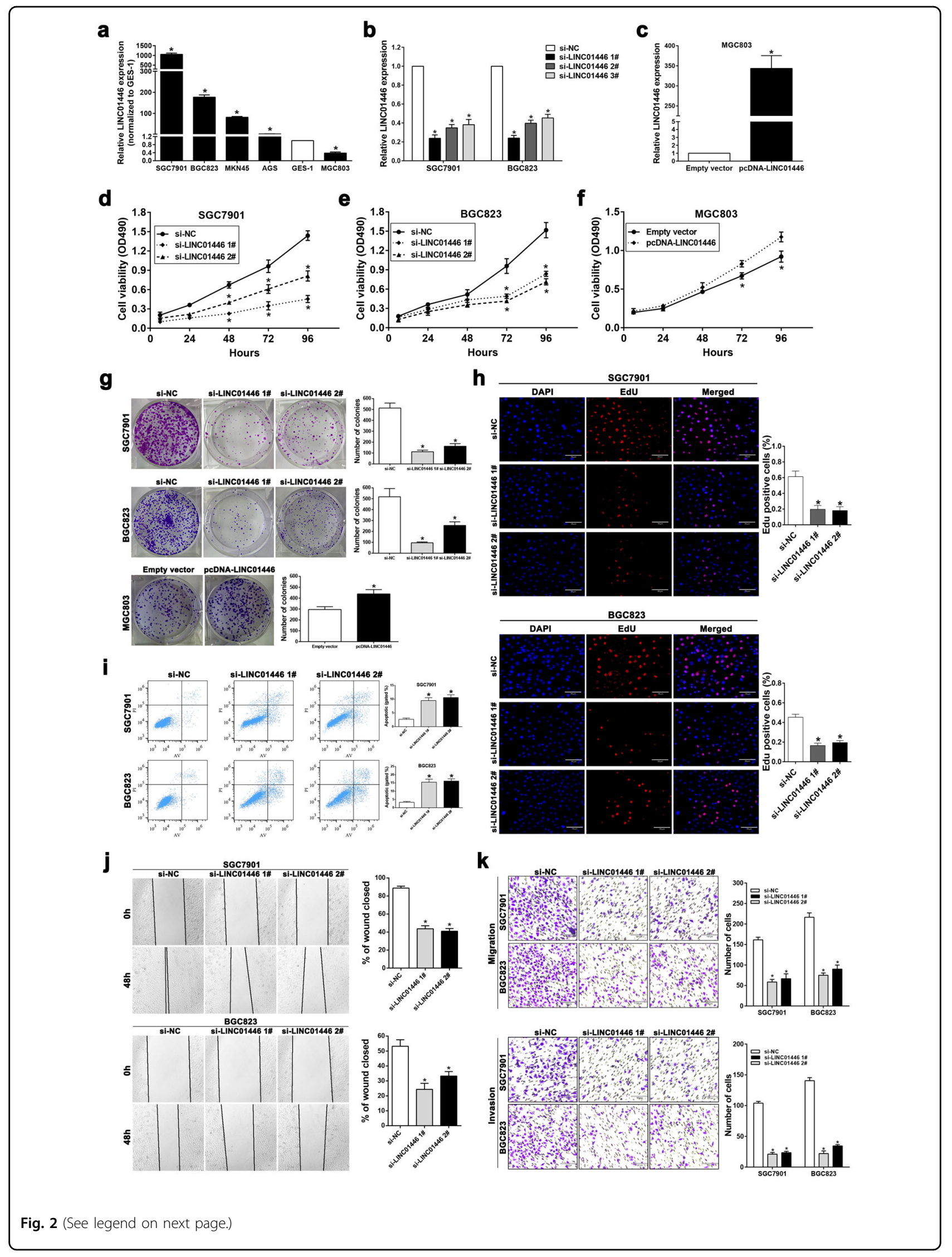


(see figure on previous page)

Fig. 2 LINC01446 facilitates the proliferation and migration of GC cells in vitro. a LINC01446 mRNA expressions in five GC and GES-1 cells were measured. b, c LINC01446 knockdown or overexpression efficiencies in GC cells were assessed. d, e MTT assay for the si-LINC01446- and si-NCtransfected SGC7901 and BGC823 cells. $\mathbf{f}$ MTT assays were conducted in the LINC01446-overexpressed MGC803 cells. $\mathbf{g}$ The proliferation of the transfected SGC7901 and BGC823 cells with si-LINC01446 or the MGC803 cells overexpressed by plasmid were detected by using colony formation assay. $\mathbf{h}$ Representative images (left) and quantification (right) for EdU immunofluorescence staining in the si-LINC01446- and si-NC-transfected SGC7901 and BGC823 cells. Scale bar: $70 \mu \mathrm{m}$. i Apoptosis in the si-LINC01446- and si-NC-transfected SGC7901 and BGC823 cells was determined using flow cytometry. $\mathbf{j}$ The migration of the LINC01446-silenced and control cells was investigated using wound-healing assay. $\mathbf{k}$ The migratory and invasive abilities of LINC01446-silenced and control cells were detected using transwell assay. Scale bar: $120 \mu m$. Data were shown as mean \pm SD, $n=3$. Student's $t$ test. ${ }^{*} P<0.05$. n.s. not significant.

a

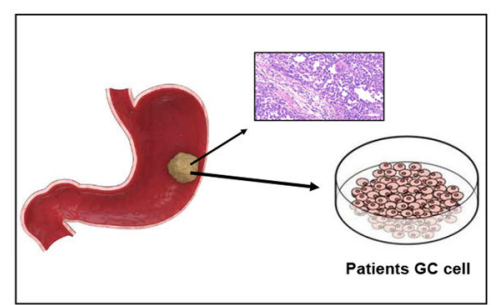

b

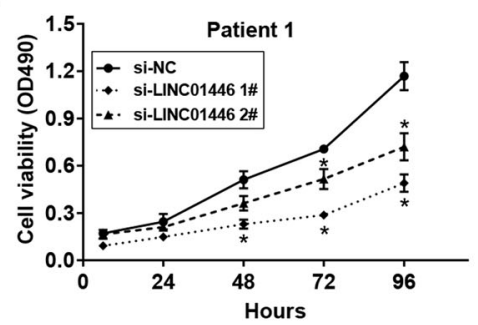

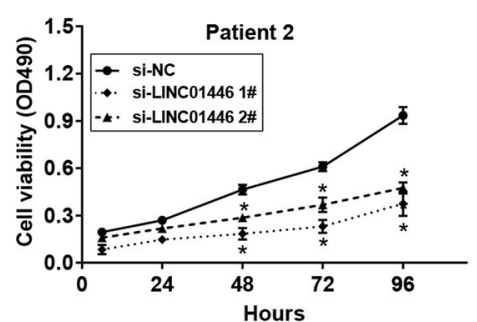

d

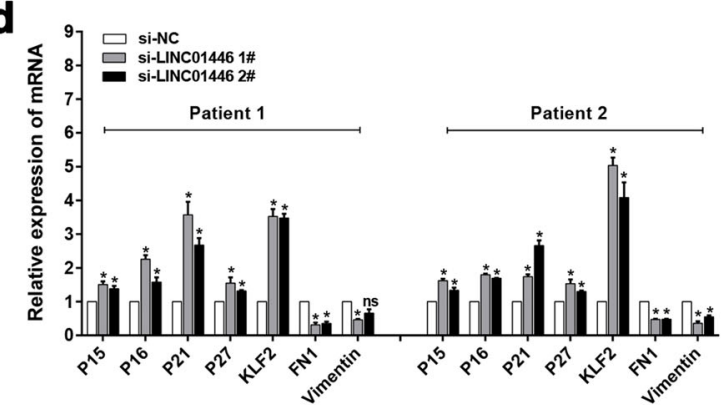

Fig. 3 Primary GC cells confirmed the anticancer effect by LINC01446 silencing. a GC tissues from two patients were obtained from the hospital, we isolated primary GC cells by collagenase digestion. b MTT assays for the si-LINC01446- or si-NC-transfected primary GC cells from patient 1 and patient 2. c The migratory abilities of LINC01446-silenced and control cells were detected using transwell assay. Scale bar: 120 m. d P15, P16, P21, p27, KLF2, FN1, and Vimentin mRNA expression levels were determined by qRT-PCR following LINC01446 knockdown. Data were shown as mean $\pm \mathrm{SD}, n=3$. Student's $t$ test, ${ }^{*} P<0.05$. n.s. not significant.

the proliferation and metastasis of tumor cells (such as cell cycle, ECM-receptor interaction, and VEGF signaling pathways) (Figs. 6c and S3). In addition, 1700 genes were differentially expressed (upregulated and downregulated genes) in SGC7901 cells after LINC01446 knockdown (fold change >1) (Fig. 7a). Next, we continued to investigate the specific LINC01446 target that leads to the observed GC cell phenotypes. As expected, several genes associated with the proliferation and metastasis of GC cells were screened and identified by qRT-PCR analysis after LINC01446 and LSD1 knockdowns (Fig. 7b-f). Among these altered genes, RASD1 has been identified as a novel tumor suppressor that regulates the proliferation, apoptosis, and metastasis of cancer cells ${ }^{19,20}$. Therefore, the RASD1 was chosen for the subsequent analyses. Western blot analysis demonstrated that LINC01446 knockdown significantly upregulated RASD1 in both SGC7901 and BGC823 cells (Fig. 7g). Next, two pairs of primers $(2000 \mathrm{bp})$ across the promoter region of RASD1 were designed to perform the chromatin immunoprecipitation (ChIP) assay and investigate the related regulatory mechanism. Our results demonstrated that LSD1 directly bound to the RASD1 promoter region-mediated demethylated H3K4, while LINC01446 interference reduced this bind and promoted the modification in GC cells (Fig. 7h, i). Taken together, our data indicated that RASD1 might be a downstream target gene of LINC01446, and LINC01446 could recruit LSD1 into RASD1 promoter, thereby resulting in epigenetic suppression through demethylated H3K4me2. 
a

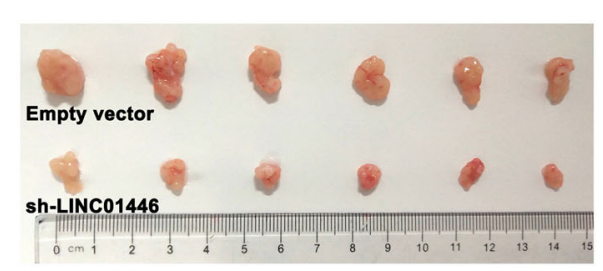

b

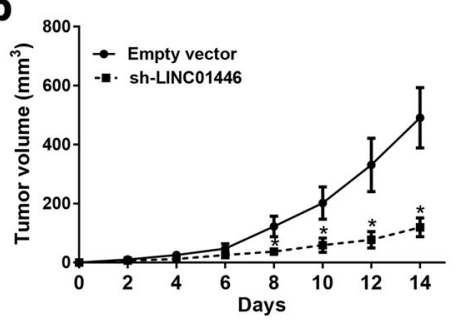

d

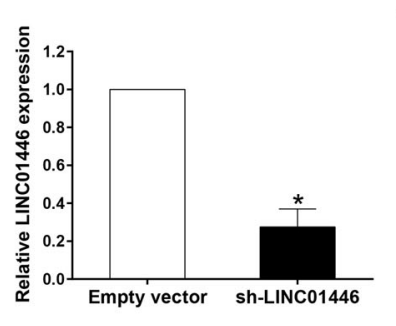

f

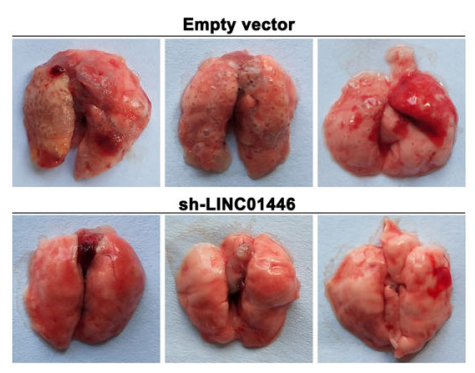

e

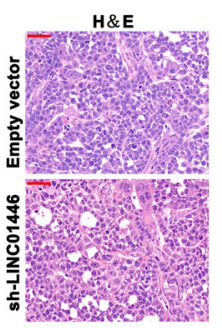

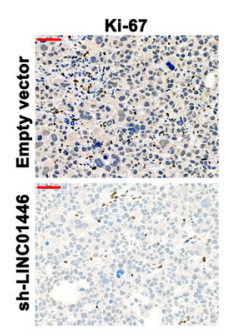

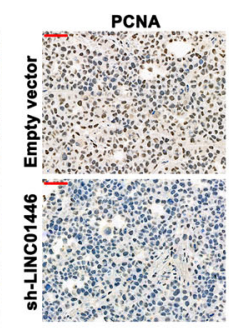

h

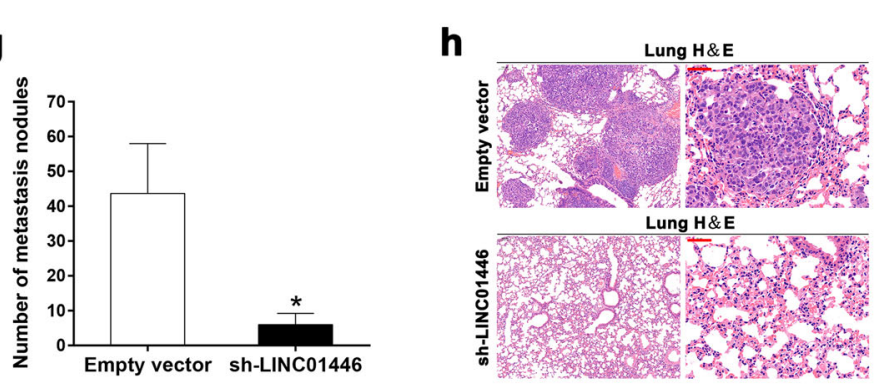

I

g

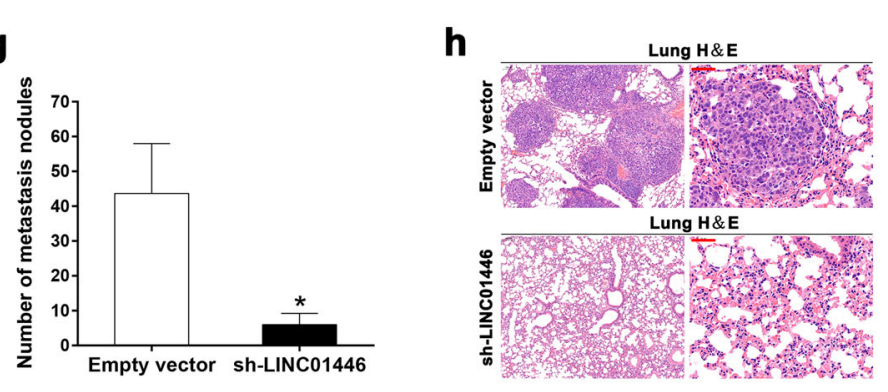

c

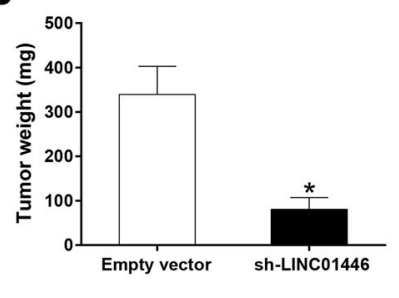

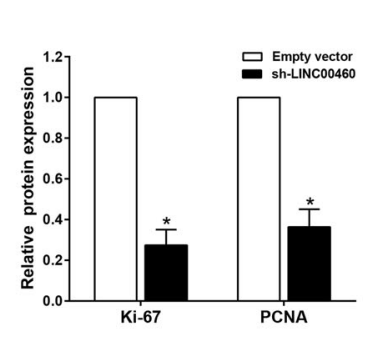

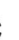
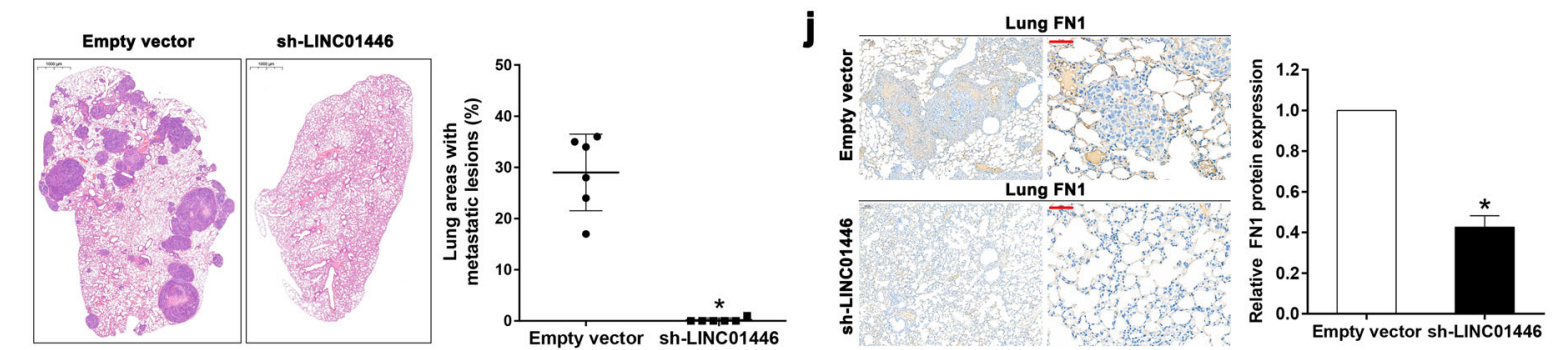

Fig. 4 Effects of LINC01446 on the tumor growth and metastasis of GC in vivo. a The transfected SGC7901 and BGC823 cells with empty vector or sh-LINC00707 were inoculated into nude mice. b Tumor volume was determined after injecting every 2 days. $\mathbf{c}$ Tumors were weighted following removing. $\mathbf{d}$ The mRNA expressions of LINC01446 in xenograft tumors were measured. e Tumor sections were stained by H\&E and IHC using anti-Ki67 and anti-PCNA antibodies. The representative staining images were shown. Scale bar: $50 \mu \mathrm{m}$. $\mathbf{f}$ The representative images for the entire lung of mice from each group were shown. $\mathbf{g}$ The numbers of metastasis nodules on the surface of lungs were counted. $\mathbf{h}$ Lung sections were stained by H\&E. Scale bar: $50 \mu \mathrm{m}$. $\mathbf{i}$ The percentage of lung areas with metastatic lesions was calculated from all mice in each group. Scale bar: $1000 \mu \mathrm{m}$. $\mathbf{j}$ Lung sections were stained by IHC with anti-FN1 antibody. The representative staining images were shown. Scale bar: $50 \mu \mathrm{m}$. Data were shown as mean \pm SD. Student's $t$ test. ${ }^{*} P<0.05$. n.s. not significant.

\section{LINC01446 plays oncogenic roles through partly suppressing RASD1 expression}

To evaluate whether RASD1 functions as a tumor suppressor in GC, we firstly measured the RASD1 mRNA expression according to the TCGA database. Our data demonstrated that the RASD1 mRNA expression significantly reduced in GC tissues relative to the normal tissues (Fig. 8a, b). Furthermore, immunohistochemical staining assay was also employed to measure the RASD1 expression in 25 matched GC and normal tissues. The representative images were presented in Fig. 8c, d, suggesting that RASD1 was downregulated in GC tissues and was negatively related to the LINC01446 level. Further functional trials demonstrated that RASD1 overexpressing could significantly suppress the proliferation and migration of GC cells (Fig. 8e-h). Next, EdU and colony formation assays were carried out to evaluate whether LINC01446 could regulate the proliferation and 


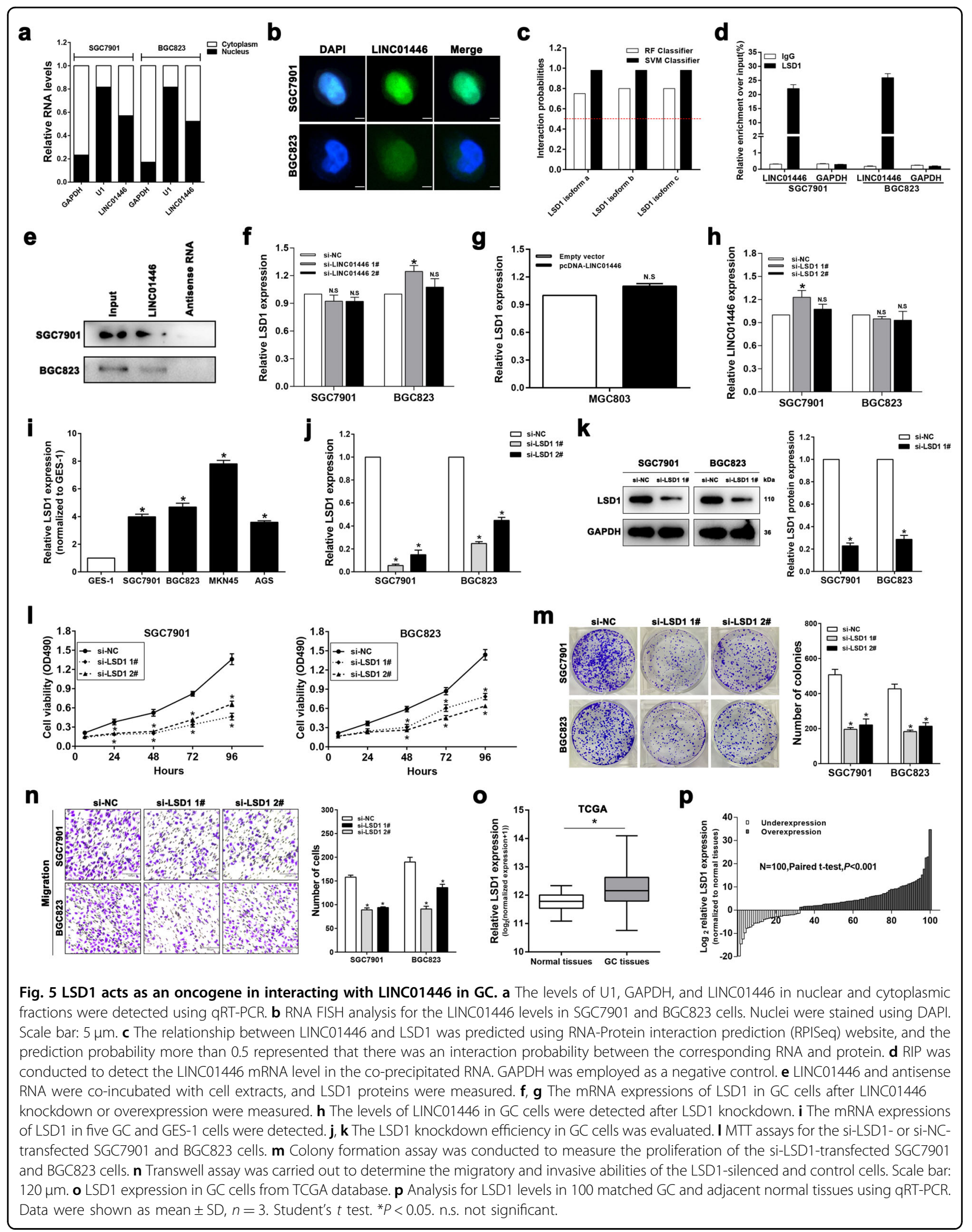



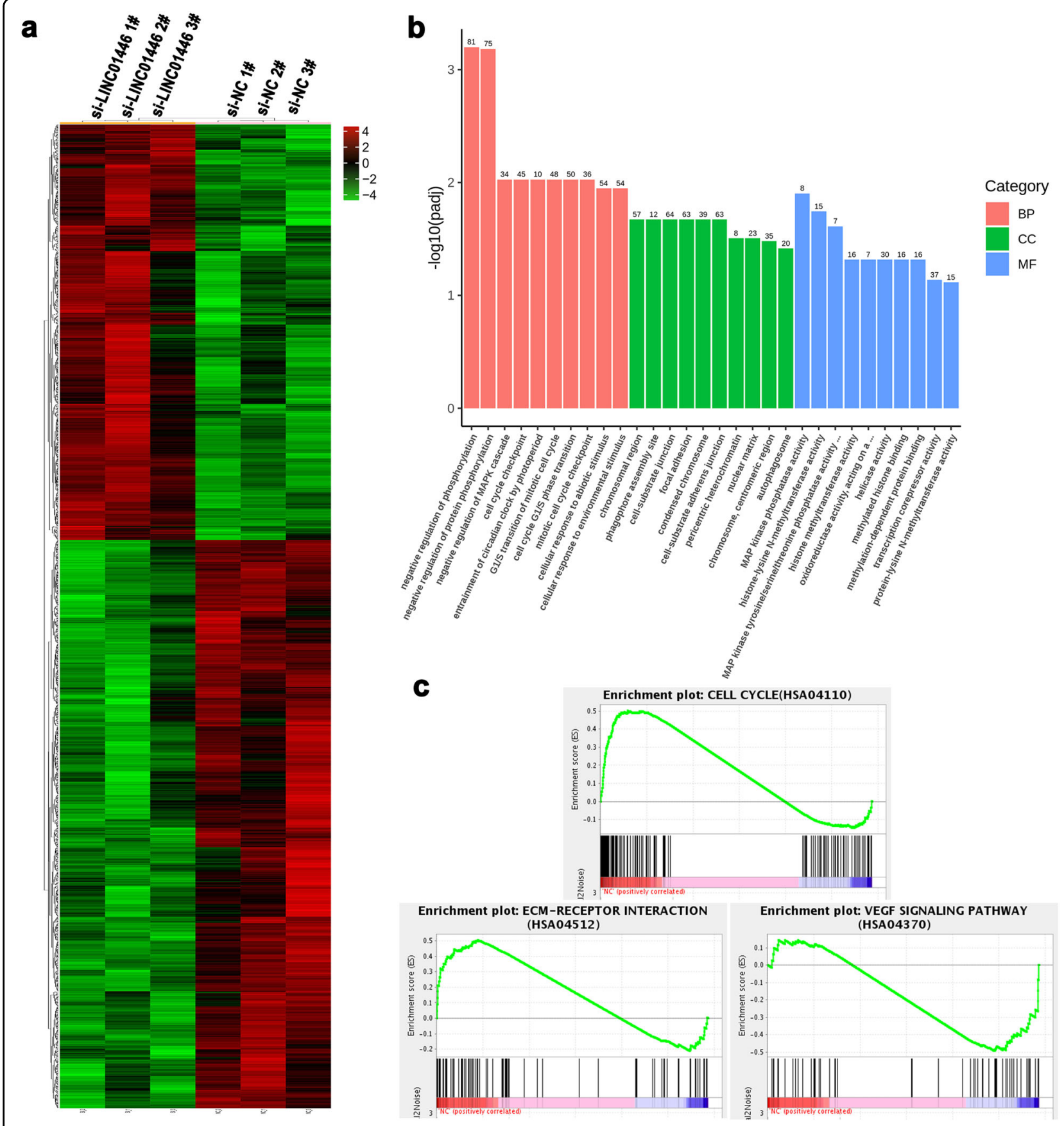

Fig. 6 RNA-seq analysis for SGC7901 cells after LINC01446 knockdown. a Mean-centered, hierarchical clustering of 1700 altered genes (FC > 1) in si-NC- and si-LINC01446-treated cells in triplicate. $\mathbf{b}$ GO analysis for all altered genes. c GSEA showed that the proliferation and metastasis-related biological functions were enriched in responses to high LINC01446 expression in SGC7901 cells based on the RNA-seq analysis after LINC01446 knockdown.

migration of GC cells by suppressing RASD1 expression. Our data proved that the co-transfection partly reversed the si-LINC01446-impaired proliferation of SGC7901 and BGC823 cells (Fig. 8i, j). Furthermore, wound-healing and transwell analyses demonstrated that RASD1 knockdown could alleviate the si-LINC01446-impaired migration and invasion of SGC7901 and BGC823 cells (Figs. S4a-e and $8 \mathrm{k})$. Taken together, our data indicated that LINC01446 might promote the proliferation and metastasis of GC cells through partly decreasing RASD1 expression. 


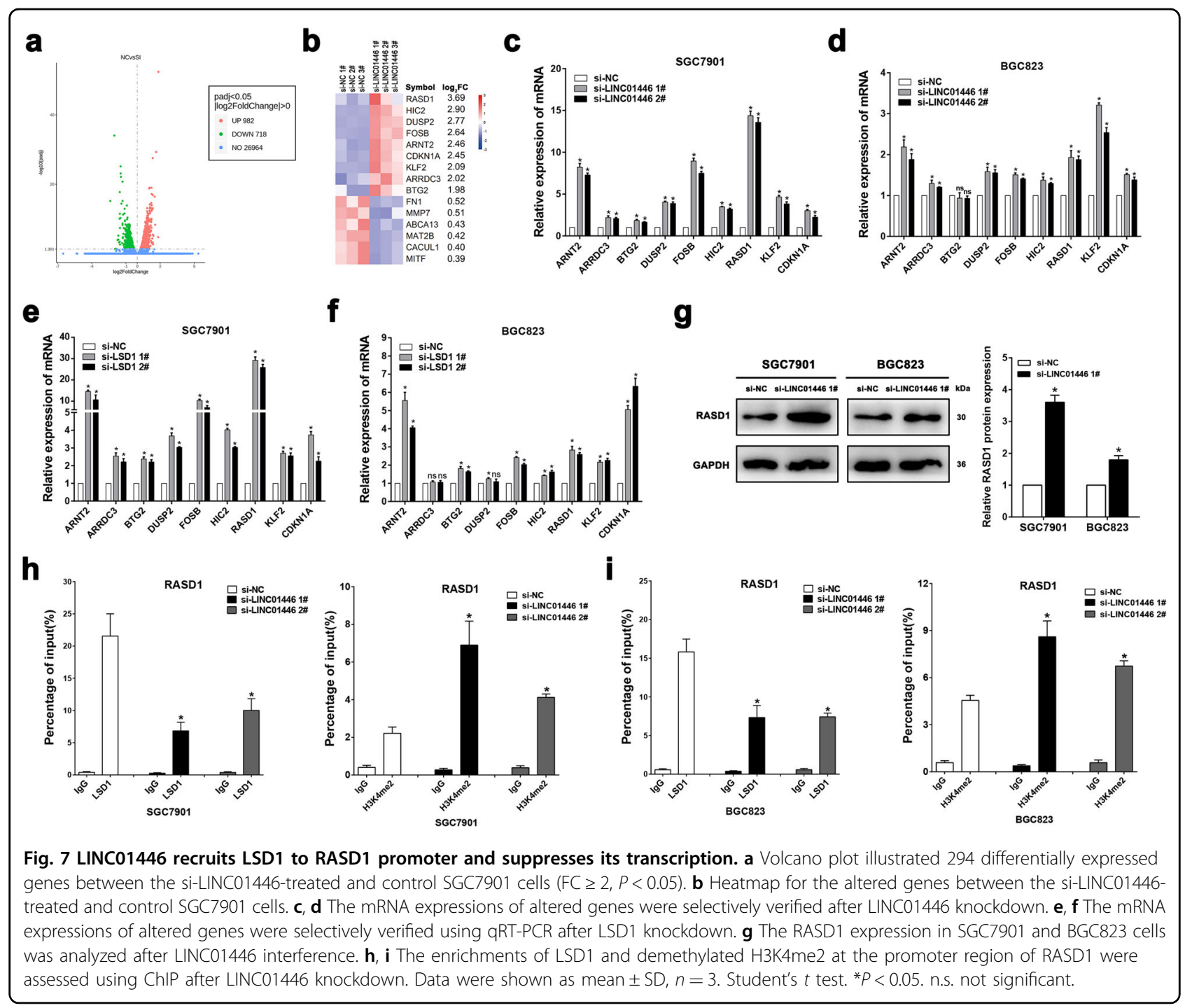

\section{Discussion}

GC has always been a severe health threat worldwide especially in the highly endemic region, China ${ }^{21,22}$. Therefore, there is an urgent need to discover clinically risk factors and relevant prognostic biomarkers, thereby developing novel precise therapies. Previous studies almost paid attentions to the effects the protein-coding genes exhibited on disease pathogenesis ${ }^{23}$. Recent advances in high-throughput RNA sequencing showed the widespread expressions of lncRNAs in various cancers $^{24-26}$. Massive evidences indicated that lncRNAs, once regarded as 'junk RNA', played distinct roles in the occurrence and progression of cancers, but the lncRNAs specifically related to GC are still not clear ${ }^{27,28}$. Therefore, LINC01446 expressions in GC tissues and cells were detected in our study. And the results indicated that the increased LINC01446 facilitated the proliferation, migration, and invasion of GC cells, which is consistent with previous studies showing that LINC01446 functions as an oncogene in glioblastoma ${ }^{14}$. Accordingly, the overexpression of LINC01446 in GC patients was positively associated with advanced TNM stage and distal metastasis. Furthermore, high LINC01446 expression in GC tissues was associated with the poor prognosis and could be an independent prognostic indicator. These results indicated that LINC01446 might serve as a clinically significant prognostic biomarker for GC and might play an important role in GC progression. So, there is an urgent need to investigate the action mechanism of LINC01446 facilitating the proliferation and metastasis of GC.

In glioblastoma, it is demonstrated that LINC01446 could promote cell proliferation and migration by competing with miRNAs and acting as ceRNAs ${ }^{14}$. In our study, LSD1 was found to be an RNA binding protein to LINC01446 and directly interacted with LINC00460, thereby affecting the proliferation, migration, and invasion 


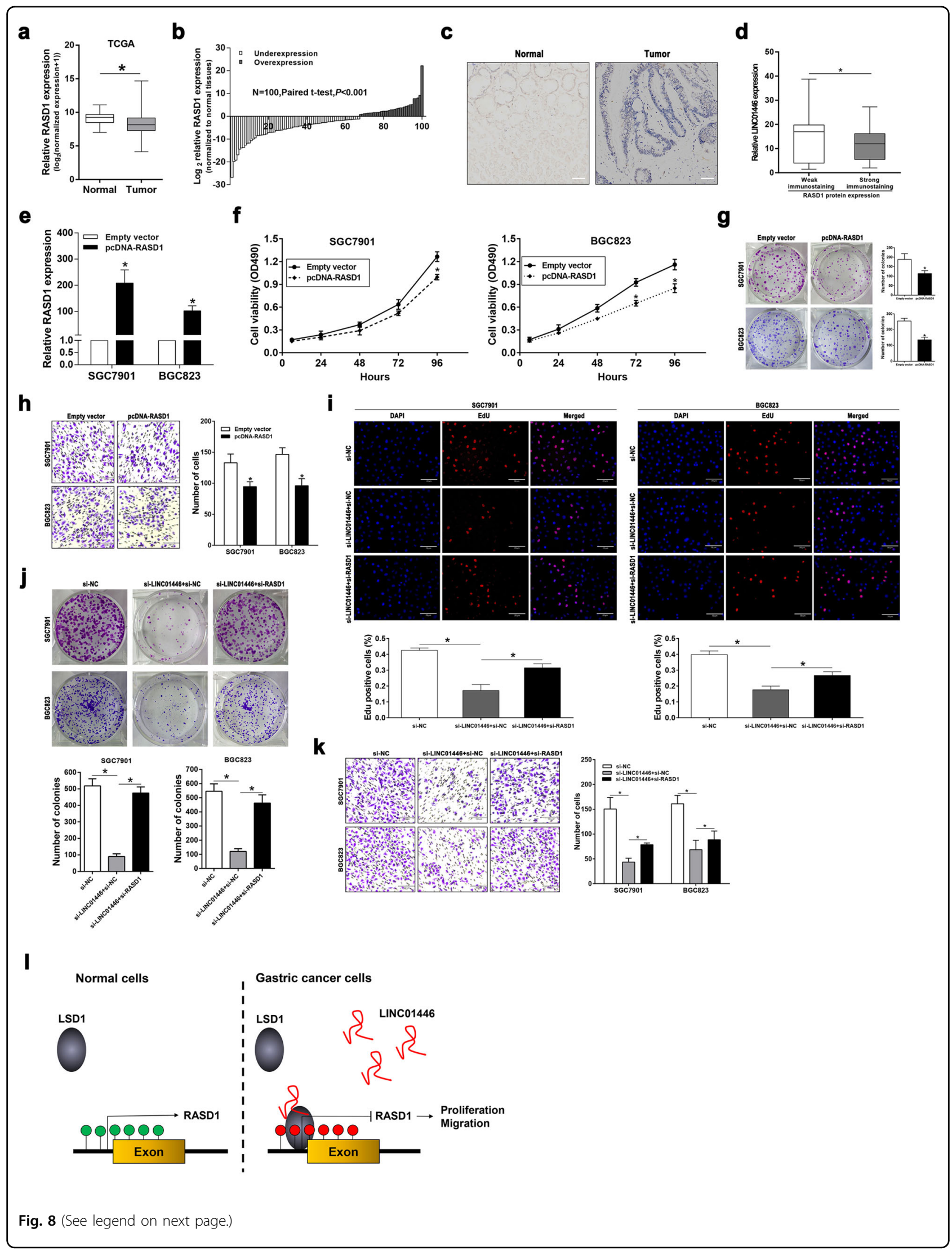


(see figure on previous page)

Fig. 8 LINC01446 acts as an oncogene by suppressing RASD1 expression in GC cells. a RASD1 expression in GC from TCGA database. Wilcoxon rank-sum test was adopted. b Analysis for RASD1 levels in 100 matched GC and normal tissues. Student's $t$ test was adopted. $\mathbf{c} \Perp H C$ staining was employed to measure the RASD1 expression in GC and normal tissues. Spearman's rank correlation was adopted. Scale bar: $100 \mu \mathrm{m}$. $\mathbf{d}$ The immunoreactivity of the RASD1 expression in GC tissues demonstrated a significantly negative correlation with the relative expression of LINC01446. Spearman's rank correlation was adopted. e The LSD1 knockdown efficiency in GC cells was verified using qRT-PCR. Student's $t$ test was adopted. f, $\mathbf{g}$ MTT and colony formation assays were carried out to determine the proliferation of the RASD1-overexpressed GC cells. Student's $t$ test was adopted. $\mathbf{h}$ The effects of RASD1 overexpression on the invasion of GC cells were assessed. Scale bar: $120 \mu \mathrm{m}$. Student's $t$ test was adopted. $\mathbf{i}, \mathbf{j}$ The proliferation of si-LINC01446 and/or si-RASD1-transfected SGC7901 and BGC823 cells was detected using EdU and colony formation assays. Scale bar: $70 \mu \mathrm{m}$. Student's $t$ test was adopted. $\mathbf{k}$ The migration of SGC7901 and BGC823 cells following co-transfection with si-LINC01446 and/or si-RASD1 was detected using transwell assay. Scale bar: $120 \mu \mathrm{m}$. Student's $t$ test was adopted. I Model of LINC01446 function and mechanisms during GC pathogenesis. Data were shown as mean $\pm \mathrm{SD}, n=3 .{ }^{*} P<0.05$. n.s. not significant.

of GC cells. Furthermore, it was also found that LSD1 expression markedly enhanced in GC tissues relative to the normal tissues, and LSD1 knockdown obviously impaired the proliferation and migration of GC cells. LSD1 is a member of the flavin-dependent LSD/KDM1 demethylase protein family, and is reported as a transcription repressor that can demethylate $\mathrm{H} 3 \mathrm{~K} 4 \mathrm{me} 1 / \mathrm{me}^{29-32}$. To explore the related signals and underlying targets that participate in the regulation of LINC01446-LSD1 axis in $\mathrm{GC}$ on an unbiased basis, we conducted high-throughput RNA sequencing following the knockdown of LINC01446 in SGC7901 cells. Further GO and GSEA analyses indicated that the gene expression profiles were primarily associated with proliferation and metastasis. Among these altered genes, we found that RASD1 was markedly upregulated after LINC01446 or LSD1 knockdown. Mechanistic investigations showed that LINC01446 suppressed the transcription of RASD1 by the LSD1-mediated H3K4 demethylation, which suggests that RASD1 is a downstream target gene of LINC01446-LSD1 axis.

RASD1 belonged to the Ras family is originally regarded as a dexamethasone-inducible gene, and its product is a receptor-independent activator of G-protein signal ${ }^{33,34}$. Although there are still some controversies regarding the roles RASD1 played in carcinogenesis, many studies revealed that RASD1 could serve as a tumor suppressor in diverse malignancies ${ }^{35-38}$. For instance, Gao et al. reported that the overexpression of RASD1 in glioma cells possibly played inhibitory roles in the migration and invasion of tumor cells through inactivating AKT/mTOR signal $^{39}$. Furthermore, Govindan et al. found that restoring RASD1 expression in three different cancer cells played a significant anti-growth role ${ }^{19}$. In our current work, RASD1 was obviously downregulated in GC tissues. Functional studies demonstrated that RASD1 could also suppress the proliferation and migration of GC cells. Interestingly, our rescue experiments illustrated that LINC01446 facilitated the proliferation and metastasis of GC partly depending on the suppress of RASD1.

In conclusions, we identified a novel GC-related IncRNA, LINC01446, and firstly demonstrated that
LINC01446 could promote the proliferation and metastasis of GC through the epigenetic knockdown of RASD1 via binding to LSD1 (Fig. 81). In addition, LINC01446 might serve as an underlying prognostic biomarker for GC. These results will provide insights into the development of novel therapies for GC.

\section{Materials and methods \\ Ethics}

The experimental protocols for the collection and application of human samples were approved by the Ethics Committee of Zhongshan Hospital, Xiamen University (Xiamen, China). All subjects provided written informed consent for surgery. All animal trials were carried out based on the national and international guidelines and approved by the Animal Care and Use Institutional Review Board of Xiamen University.

\section{Collection of human samples}

After surgery, $100 \mathrm{GC}$ and normal gastric epithelial tissues were immediately harvested from the GC patients at Zhongshan Hospital, Xiamen University and Second Affiliated Hospital, Nanjing Medical University (Nanjing, China). The clinicopathological information about all subjects were acquired according to the patient records, which were listed in Tables 1 and 2.

\section{Quantitative real-time PCR (qRT-PCR)}

In brief, total RNA was firstly extracted from GC cells with TRIzol (Invitrogen). Next, cDNA was synthesized with $1 \mu \mathrm{g}$ total RNA based on the protocols of kit (Takara). Finally, GAPDH was employed as a negative control to determine the relative mRNA level of target genes in Quant Studio 6 Flex system (Applied Biosystems). All of the primer sequences were listed in Table S1.

\section{Cell culture and transfection}

A normal human gastric epithelium cell line (GES-1) and five GC cell lines including SGC7901, BGC823, MGC803, MKN45, and AGS without mycoplasma were provided by Chinese Academy of Sciences (Shanghai, 
China). SGC7901, BGC823, and MGC803 cells were grown in RPMI-1640 (Gibco) medium; SGC7901, AGS, and GES-1 cells were incubated in DMEM (Gibco) containing 10\% fetal bovine serum (FBS; Gibco), $100 \mathrm{U} / \mathrm{ml}$ penicillin and $100 \mathrm{mg} / \mathrm{ml}$ streptomycin (Invitrogen) at $37^{\circ} \mathrm{C}$ with $5 \% \mathrm{CO}_{2}$.

Lipofectamine 2000 (Invitrogen) was used to transfect si-LINC01446, si-LSD1, si-RASD1, and negative control siRNA (si-NC). The transfections of pcDNA-LINC01446, pcDNA-RASD1, and empty vectors were performed by using X-tremeGENE HP DNA (Roche). After 24 or $48 \mathrm{~h}$ of transfections, the cells were harvested. All of the above siRNA sequences were listed in Table S3.

\section{Cell proliferation and migration assays}

MTT method was firstly used to detect cell viability thereby assessing the cell proliferation. In brief, the transfected SGC7901 and BGC823 cells (3000 cells/well) with si-LINC01446 or pcDNA-LINC01446 were seeded in 96-well plates. Next, cell viability was detected every $24 \mathrm{~h}$ based on MTT kit protocols. For colony formation assay, 400 cells were inoculated into sixwell plates and cultured at $37^{\circ} \mathrm{C}$ for 14 days. Then, the colonies were fixed with methanol and stained with hematoxylin. For EdU assay, a 5-ethynyl-2-deoxyuridine detection kit (Ribobio) was employed, and DAPI was employed to label the nuclei of GC cells. Finally, the EdU-positive cells were calculated in five randomly selected fields per well under a fluorescent microscopy. All trials were conducted in triplicate.

Wound-healing assay was carried out to measure cell migration. In brief, cells were firstly inoculated in sixwell plates. After $24 \mathrm{~h}$ of culture with serum-free medium, the monolayer cells were linearly scraped to generate an artificial wound. For the transwell migration and invasion assays, transwell chambers (Corning) with a membrane pore size of $8 \mu \mathrm{m}$ were coated without or with Matrigel (BD Biosciences). A total of $5 \times 10^{4}$ or $1 \times 10^{5}$ cells were inoculated in the upper chambers and cultured in the medium supplemented with $1 \%$ FBS. Then, the lower chamber was filled using the medium with $10 \%$ FBS. After 12 or $24 \mathrm{~h}$ of incubation, the cells were fixed, stained, and counted in an inverted microscope. All trials were conducted in triplicate.

\section{Flow cytometry analysis for apoptosis}

After $48 \mathrm{~h}$ of transfection with si-LINC01446 or si-NC, SGC7901, and BGC823 cells were harvested. Next, these cells were stained based on the protocols of a FITC Annexin V Apoptosis Detection kit (BD Biosciences), and were subsequently analyzed using flow cytometer (FACScan ${ }^{\circledR}$ ) and CellQuest software (BD Biosciences). Finally, the cells were assigned into living, dead, early, and late apoptotic cells, and the percentage of early apoptotic cells was calculated using the control cells in each trial.

\section{In vivo assays for the growth and metastasis of tumors}

For the tumorigenicity assay, the stably transfected SGC7901 and BGC823 cells $\left(2 \times 10^{6}\right.$ cells $)$ with shLINC01446 or empty vectors were firstly injected into the abdominal cavities of 4 weeks of male $\mathrm{BALB} / \mathrm{c}$ nude mice $(n=6)$. At the end of this trial, all the mice were sacrificed, and their tumor tissues were weighed and stored for further experiments. In addition, according to the previous report ${ }^{40}$, tumor volume $\left(\mathrm{mm}^{3}\right)$ was calculated by using its longest and shortest diameters.

To evaluate the effects of LINC01446 on tumor metastasis, the stably transfected SGC7901 cells $\left(2 \times 10^{6}\right.$ cells) with sh-LINC01446 or empty vectors were firstly injected into the tail veins of 4 weeks of male BALB/c nude mice $(n=6)$. After 6 weeks, all mice were sacrificed and then their lungs were removed for hematoxylin and eosin (H\&E) staining. Finally, the numbers of metastatic tumors in the lungs were observed and calculated in a blinded manner.

\section{Subcellular fractionation and fluorescent in situ hybridization (FISH)}

A PARIS kit (Life Technologies) was used to separate nuclear and cytoplasmic RNAs for qRT-PCR analysis. All of the primer sequences were listed in Table S3. For FISH assay, GC cells, and tissues were firstly fixed with $4 \%$ formaldehyde for $15 \mathrm{~min}$ after washing with PBS. Then, the fixed cells were incubated in $1 \%$ pepsin and dehydrated using $70 \%, 90 \%$, and $100 \%$ ethanol, respectively. Next, the cells were air dried and co-incubated with $40 \mathrm{nM}$ FISH probe diluted in hybridization buffer at $80^{\circ} \mathrm{C}$ for $2 \mathrm{~min}$. The hybridization assay was conducted at $55^{\circ} \mathrm{C}$ for $2 \mathrm{~h}$, and then the slides were washed and dehydrated. Finally, the slides were air dried and then mounted with Prolong Gold Antifade Reagent containing DAPI for further analysis. RNA FISH probe was synthesized by Bogu Co., Ltd. (Shanghai, China), and its sequences were presented in Table S3.

\section{RNA immunoprecipitation (RIP) and RNA pull-down assays}

RIP was carried out based on the protocols of an EZMagna RIP kit (Millipore). In brief, when SGC7901 and BGC823 cells influence reached at $90 \%$, all cells were scraped off and were subsequently lysed using lysis buffer. Next, $100 \mu \mathrm{L}$ cell extracts were co-incubated with the magnetic beads conjugated with anti-LSD1 or control IgG antibodies (Millipore) at $4{ }^{\circ} \mathrm{C}$ for $6 \mathrm{~h}$. Subsequently, the complexes were treated with $0.1 \%$ SDS or $0.5 \mathrm{mg} / \mathrm{ml}$ proteinase $\mathrm{K}$ at $55^{\circ} \mathrm{C}$ for $30 \mathrm{~min}$ to remove proteins following washing with the washing buffer. Finally, a NanoDrop spectrophotometer (Thermo Scientific) and bioanalyzer (Agilent) were used to measure the concentration and quality of RNA. The purified immunoprecipitated RNA was used for further qRT-PCR analysis. 
For the RNA pull-down assay, LINC01446 was transcribed with T7 RNA polymerase (Ambio Life) in vitro, purified using a RNeasy Plus Mini kit (Qiagen), and then treated using RNase-free DNase I (Qiagen). Next, the transcribed LINC01446 was labeled using the Biotin RNA Labeling Mix (Ambio Life). Finally, the RNA pull-down assay was conducted following the protocols of Pierce ${ }^{\mathrm{TM}}$ Magnetic RNA-Protein Pull-Down kit (Thermo).

\section{RNA sequencing}

SGC7901 cells were cultured into a sixwell plate and transfected with the siRNA targeting LINC01446 or negative control. After $24 \mathrm{~h}$ of transfection, the transfected cells were harvested for RNA extraction, followed by subsequent library construction, and sequencing. RNA sequencing was performed by Novogene, and the raw data could be accessed in the NCBI SRA database (SRA ID: SUB6506100).

\section{Chromatin immunoprecipitation (ChIP) assay}

ChIP assay was conducted based on the protocols of an EZ-ChIP Chromatin Immunoprecipitation kit (Millipore). In brief, sonication was used to proceed the cross-linked chromatin DNA into the 200-500 bp of fragments. Then, the chromatin was immunoprecipitated with the corresponding antibodies. Finally, the isolated DNA was evaluated using qRT-PCR analysis.

Western blotting and immunocytochemistry (IHC) analysis

Western blotting and $\mathrm{IHC}$ analysis were conducted based on the previously reported procedures ${ }^{40}$.

\section{Statistical analysis}

All trials were independently conducted at least three times, with the samples in triplicate. All statistical analyses were carried out with SPSS 22.0 and Prism 5.0 (GraphPad) software. Then, two-tailed Student's $t$ test was used for the assessment of statistical difference between two groups, and Pearson correlation analysis was employed to evaluate the interactions among gene sets. Data were shown as mean $\pm \mathrm{SD}$. ${ }^{*} P<0.05$ was regarded as significance.

\section{Acknowledgements}

This study was supported by the National Natural Science Foundation of China (grant code: 81770558, 81370591) and the Natural Science Foundation of Fujian Province (grant code: 2019J01554). The funders had no role in study design, data collection, data analysis, interpretation, and writing of the report. The corresponding authors (H.X., J.R., and C.L.) had full access to all of the data in the study and had final responsibility for the decision to submit for publication.

\footnotetext{
Author details

'Department of Gastroenterology, Zhongshan Hospital, Xiamen University, Xiamen, Fujian, PR China. ${ }^{2}$ Institute for Microbial Ecology, School of medicine, Xiamen University, Xiamen, Fujian, PR China. ${ }^{3}$ Department of Obstetrics and Gynecology, The First Affiliated Hospital of Xiamen University, Xiamen, Fujian,
}

PR China. ${ }^{4}$ Department of Breast Surgery, Quanzhou First Hospital Affiliated to Fujian Medical University, Quanzhou, Fujian, PR China. ${ }^{5}$ Department of Gynecology, The First Affiliated Hospital of Nanjing Medical University, Nanjing, Jiangsu, PR China

\section{Competing Interests}

The authors declare no competing interest.

\section{Publisher's note}

Springer Nature remains neutral with regard to jurisdictional claims in published maps and institutional affiliations.

Supplementary Information accompanies this paper at (https://doi.org/ 10.1038/s41419-020-2729-0).

Received: 4 February 2020 Revised: 12 May 2020 Accepted: 13 May 2020 Published online: 10 July 2020

References

1. Chen, W. et al. Cancer statistics in China, 2015. CA Cancer J. Clin. 66, 115-132 (2016).

2. Bray, F. et al. Global cancer statistics 2018: GLOBOCAN estimates of incidence and mortality worldwide for 36 cancers in 185 countries. CA Cancer J. Clin. 68, 394-424 (2018).

3. Li, T. T. et al. Prognostic and predictive blood biomarkers in gastric cancer and the potential application of circulating tumor cells. World J. Gastroenterol. 24, 2236-2246 (2018).

4. Van Cutsem, E., Sagaert, X., Topal, B., Haustermans, K. \& Prenen, H. Gastric cancer. Lancet 388, 2654-2664 (2016).

5. Vedeld, H. M., Goel, A. \& Lind, G. E. Epigenetic biomarkers in gastrointestinal cancers: The current state and clinical perspectives. Semin. Cancer Biol. 51, 36-49 (2018).

6. Mattick, J. S. \& Rinn, J. L. Discovery and annotation of long noncoding RNAs. Nat. Struct. Mol. Biol. 22, 5-7 (2015).

7. Xu, T. P. et al. KLF5 and MYC modulated LINC00346 contributes to gastric cancer progression through acting as a competing endogeous RNA and indicates poor outcome. Cell Death Differ. 26, 2179-2193 (2019).

8. YiRen, $\mathrm{H}$. et al. Long noncoding RNA MALAT1 regulates autophagy associated chemoresistance via miR-23b-3p sequestration in gastric cancer. Mol. Cancer 16, 174 (2017).

9. Wang, X. et al. C8orf76 promotes gastric tumorigenicity and metastasis by directly inducing IncRNA DUSP5P1 and associates with patient outcomes. Clin. Cancer Res. 25, 3128-3140 (2019).

10. Sakai, S. et al. Long noncoding RNA ELIT-1 acts as a Smad3 cofactor to facilitate TGFbeta/Smad signaling and promote epithelial-mesenchymal transition. Cancer Res. 79, 2821-2838 (2019).

11. Yang, X. Z. et al. LINC01133 as ceRNA inhibits gastric cancer progression by sponging miR-106a-3p to regulate APC expression and the Wnt/beta-catenin pathway. Mol. Cancer 17, 126 (2018).

12. Zhuo, W. et al. Long noncoding RNA GMAN, up-regulated in gastric cancer tissues, is associated with metastasis in patients and promotes translation of Ephrin A1 by competitively binding GMAN-AS. Gastroenterology 156, 676-691 (2019).

13. Zhang, E. et al. A novel long noncoding RNA HOXC-AS3 mediates tumorigenesis of gastric cancer by binding to YBX1. Genome Biol. 19, 154 (2018).

14. Zhang, L. et al. LncRNA LINC01446 promotes glioblastoma progression by modulating miR-489-3p/TPT1 axis. Biochem. Biophys. Res. Commun. 503, 1484-1490 (2018).

15. Miao, $\mathrm{H}$. et al. A long noncoding RNA distributed in both nucleus and cytoplasm operates in the PYCARD-regulated apoptosis by coordinating the epigenetic and translational regulation. PLoS Genet. 15, e1008144 (2019).

16. Xia, C., Fan, J., Emanuel, G., Hao, J. \& Zhuang, X. Spatial transcriptome profiling by MERFISH reveals subcellular RNA compartmentalization and cell cycledependent gene expression. Proc. Natl Acad. Sci. USA 116, 19490-19499 (2019).

17. Fu, X., Zhang, P. \& Yu, B. Advances toward LSD1 inhibitors for cancer therapy. Future Med. Chem. 9, 1227-1242 (2017). 
18. Li, Y. et al. HBXIP and LSD1 scaffolded by IncRNA hotair mediate transcriptional activation by c-Myc. Cancer Res. 76, 293-304 (2016).

19. Vaidyanathan, G. et al. The Ras-related protein AGS1/RASD1 suppresses cell growth. Oncogene 23, 5858-5863 (2004).

20. Tang, Y. J. et al. Tracing tumor evolution in sarcoma reveals clonal origin of advanced metastasis. Cell Rep. 28, 2837-2850 (2019).

21. Zhang, $X$. et al. Endoscopic screening in Asian countries is associated with reduced gastric cancer mortality: a meta-analysis and systematic review. Gastroenterology 155, 347-354 (2018).

22. Liu, K. Qin, M. \& Huang, J. The prescreening tool for gastric cancer in China. Gut. https://doi.org/10.1136/gutjnl-2019-319591 (2019).

23. Sullenger, B. A. \& Nair, S. From the RNA world to the clinic. Science $\mathbf{3 5 2}$ 1417-1420 (2016).

24. Huarte, M. The emerging role of IncRNAs in cancer. Nat Med 21, 1253-1261 (2015).

25. Kopp, F. \& Mendell, J. T. Functional classification and experimental dissection of long noncoding RNAs. Cell 172, 393-407 (2018).

26. Ramnarine, V. R. et al. The evolution of long noncoding RNA acceptance in prostate cancer initiation and progression, and its clinical utility in disease management. EUR UROL (2019).

27. Arun, G., Diermeier, S. D. \& Spector, D. L. Therapeutic targeting of long noncoding RNAs in cancer. Trends Mol. Med. 24, 257-277 (2018).

28. Tam, C. et al. LncRNAs with miRNAs in regulation of gastric, liver, and colorectal cancers: updates in recent years. Appl. Microbiol. Biotechnol. 103 4649-4677 (2019).

29. Majello, B., Gorini, F., Sacca, C. D. \& Amente, S. Expanding the role of the histone lysine-specific demethylase LSD1 in cancer. Cancers 11, 324 (2019).

30. Byun, S. et al. A postprandial FGF19-SHP-LSD1 regulatory axis mediates epigenetic repression of hepatic autophagy. EMBO J. 36, 1755-1769 (2017).
31. Kim, S. A., Chatterjee, N., Jennings, M. J., Bartholomew, B. \& Tan, S. Extranucleosomal DNA enhances the activity of the LSD1/CoREST histone demethylase complex. Nucleic Acids Res. 43, 4868-4880 (2015).

32. Ambrosio, S., Sacca, C. D. \& Majello, B. Epigenetic regulation of epithelial to mesenchymal transition by the Lysine-specific demethylase LSD1/KDM1A. Biochim. Biophys. Acta Gene Regul. Mech. 1860, 905-910 (2017).

33. Nojima, M. et al. Genomic screening for genes silenced by DNA methylation revealed an association between RASD1 inactivation and dexamethasone resistance in multiple myeloma. Clin. Cancer Res. 15, 4356-4364 (2009).

34. Chen, J., Zhang, X., Wang, Y., Ye, Y. \& Huang, Z. Differential ability of formononetin to stimulate proliferation of endothelial cells and breast cancer cells via a feedback loop involving MicroRNA-375, RASD1, and ERalpha. Mol. Carcinog. 57, 817-830 (2018).

35. Tian, J. et al. Calycosin inhibits the in vitro and in vivo growth of breast cancer cells through WDR7-7-GPR30 Signaling. J. Exp. Clin. Cancer Res. 36, 153 (2017).

36. O'Neill, D. et al. Development and exploitation of a novel mutant androgen receptor modelling strategy to identify new targets for advanced prostate cancer therapy. Oncotarget 6, 26029-26040 (2015).

37. Munagala, R., Aqil, F., Vadhanam, M. V. \& Gupta, R. C. MicroRNA 'signature' during estrogen-mediated mammary carcinogenesis and its reversal by ellagic acid intervention. Cancer Lett. 339, 175-184 (2013).

38. de Souza, R. S. P. et al. Epigenetically deregulated microRNA-375 is involved in a positive feedback loop with estrogen receptor alpha in breast cancer cells. Cancer Res. 70, 9175-9184 (2010).

39. Gao, S. et al. Overexpression of RASD1 inhibits glioma cell migration/invasion and inactivates the AKT/mTOR signaling pathway. Sci. Rep. 7, 3202 (2017).

40. Lian, Y. et al. A novel IncRNA, LINC00460, affects cell proliferation and apoptosis by regulating KLF2 and CUL4A expression in colorectal cancer. Mol. Ther Nucleic Acids 12, 684-697 (2018). 